\title{
Mythri 1.0-Progress of an Indian Surgical Robot
}

\author{
Vikas V1, $\quad$ Aravind Reddy Voggu ${ }^{2} \quad$ Kirit Arumalla1 \\ Anita Mahadevan ${ }^{3}$ Madhav Rao ${ }^{2, \odot}$
}

Ronak Doshi $\quad$ Aravind Ramkumar ${ }^{2}$

Address for correspondence Vikas V, MBBS, MS, MCh, DNB, PhD, Quarter No.44, Type 5, Block 3, BRC Campus, NIMHANS Residential Quarters, Dharmaram P.O., Bengaluru 560029, Karnataka, India (e-mail: vikas.drv@gmail.com).

\begin{abstract}
Keywords

- neurosurgical robot

- Mythri 1.0

- robotic microscope

- neurosurgical

microscope

Neurosurgical procedures are performed using operating microscopes. The technology of most microscopes has not changed much over the past 60 years. The National Institute of Mental Health and Neurosciences and International Institute of Information Technology based at Bengaluru have embarked on joint collaboration for developing robot for neurosurgical applications. As a working prototype, robotic microscope Mythri 1.0 has been developed. An overview of the development process, working, and features of the device is presented in the article.
\end{abstract}

\section{Introduction}

Robotics deals with the science of automatic tools designed to execute specific tasks. Neurosurgery has always been a technology-intensive specialty. Tools which increase precision and minimize risk have always been a focus of neurosurgical research. The utilization of robotics for neurosurgical applications is thus an evolutionary step. Microscopic surgery has been a fundamental tenet in the practice of neurosurgery and has stood the test of time. Microscopic surgery ensured that reliable and safe visualization of the deepest portions of the human brain is achieved and surgical maneuvers hitherto thought impossible could be performed. Surgical microscopes evolved in the west and Indian contribution to the development of surgical microscopes has been minimal. The work involving the construction of Mythri 1.0, an indigenous neurosurgical robotic microscope is the focus of this article.

\section{Collaborative Effort}

The National Institute of Mental Health and Neurosciences (NIMHANS) is a tertiary care institute dedicated to the treatment of neurosurgical disorders. The center over the past couple of decades has established itself as one of the largest centers offering neurosurgical care in the country. The International
Institute of Information Technology (IIIT)-B is an engineering institute with expertise in electronics and computer science. IIIT-B through its E-Health Research Centre which was created in 2015 has forayed into niche areas in the health care domain. Robotics for neurosurgical applications is one of the focus areas of the collaboration. The initial work has been in the field of assistive robotics and neurosurgical robots. The work has been conducted at the Surgical and Assistive Robotics Laboratory (SARL), IIIT-B.

An operating microscope is one of the most common tools used by a neurosurgeon. The designs of most contemporary microscopes have not significantly changed over the past several decades. The team attempted to redesign the operating neurosurgical microscope as an attempt to develop core competence in surgical robotics.

\section{Surgical Robotics}

Robotic surgery has established itself across several distinct surgical specialties. The maximum impact of robotic surgery has been in urologic surgery. Orthopaedic surgery is another specialty in which robotic tools are found increasing use. Though robot-assisted neurosurgery, ${ }^{1}$ especially for stereotactic applications, was demonstrated much earlier, routine use 
of robots for neurosurgical applications is yet to happen. As of now, dedicated robots are available only for restricted neurosurgical procedures.

At its heart, a surgical robot is a controllable electromechanical tool with built-in sensors and actuators. The current robotic devices used in surgical procedures are master-slave devices with no autonomous features and are more or less form an advanced surgical tool. As an engineering specialty, robotics integrates principles from diverse engineering fields including mechanical engineering, electronics and control systems, ergonomics, sensor and actuator technology, and information technology. An excellent surgical robot is one which eases the surgical task either in terms of improving accuracy, reducing complications, or reduction in skill level required for a particular surgery. As with all surgical tools, there is no recipe for an excellent surgical robot. Various surgical robots have entered the surgical field only to fall by the wayside due to multiple factors. Like any other surgical tool, acceptability among the broader surgical community determines the success of robotic surgery programs.

\section{Neurosurgical Visualization}

Very few surgical specialties have adopted microscopic visualization. Surgery on the ear, eye, and microvascular surgery performed by plastic surgeons are examples where the operative microscope is part of the surgical armamentarium. Microneurosurgery is now the standard of care for most brain and spinal cord surgeries. The operative microscopic visualization allows for accurate visualization of the compact anatomical structures within the brain and spinal cord. Together with micro-instrumentation, neurosurgery as a subject showed dramatic changes in outcomes in complex neurosurgical diseases. Safe access and intervention of structures deep in the brain were possible mainly due to advances in operative microscopic visualization. Prof. Yasargil pioneered microneurosurgery in the 1960s.

Neurosurgical operating microscopes have emerged to be the most complex operating microscopes. ${ }^{2}$ The complexity of the devices is due to various features specific to neurosurgical procedures. The neurosurgical operating field requires very high magnification. Magnification and binocular vision are prerequisites for performing surgical procedures in the deep parts of the brain. The neurosurgical operating field changes in both depth and field of view. Changes in position and magnification are thus dynamic. A "hands-free" approach to microscope manipulation has resulted in several modifications. Of interest is the mouth control system initially popularized employed by Prof. Yasargil. Foot-based control and chairs with integrated microscopic controls are surgeon user interfaces already in existence. Prof Yasargil terms the ability to generate stereoscopic images as the most critical function of the operating microscope. ${ }^{3}$ The distance between the objective lens of a contemporary microscope is approximately $16 \mathrm{~mm}$, which is adequate for most neurosurgical work.

Various aspects of microscopic visualization have been the focus of several research groups. Belykh et $\mathrm{al}^{4}$ have reported on a robotic microscope, KINEVO for neurosurgical applications. The device has several new features with novel intraoperative applications. The ViTOM $^{5-8}$ is one of the older devices which attempted to bridge the gap between microscopic and endoscopic visualization. Newer microscope tools have been developed to enhance surgical visualization such as ORBEYE, a $4 \mathrm{~K}$ three-dimensional (3D) microvideoscope 3D-Eye-Flex, BrightMatter Servo System (Synaptive Medical). ${ }^{9-14}$ Sato et al ${ }^{15}$ have reported on the use of laser as a lighting source with benefits of a decrease in the heat output and improved visualization. User interfaces for microscopes have also been the focus of some studies. ${ }^{16,17}$

Endoscopes are another set of tools to provide visualization in neurosurgical procedures. A separate development has happened in endoscope technology. ${ }^{18-20}$ Endoscopes have a single camera. Newer systems provide methods for $3 \mathrm{D}$ visualization (3D glasses). Thus, microscopes and endoscopes have had differing evolutionary paths and have different technical challenges. Devices with augmented reality capability have also been described..$^{21-24}$

\section{Device Development}

The name Mythri means "friendship" and has Sanskrit origins. The device is the first product of the robotic surgery program coming out from the collaborative effort between NIMHANS and IIIT-B. The first version, Mythri 1.0, is a working prototype and aims to provide the functionality of a conventional operating microscope. The device is a multijointed robotic device with a high range of operative mobility. The design has been such that a neurosurgeon can seamlessly shift from using a traditional microscope to the device without additional training. The breakthrough achieved in the design of Mythri 1.0 is in making spatial stabilization of the camera without a counterbalance system. Further work is required to incorporate dedicated device cameras, user interfaces, and visualization tools. The device is a fully working prototype and is a proof of concept of a hollow core hyperflexible hyperredundant robotic device indigenously developed by the team.

\section{Technical Features}

The robotic device comprises two independent systems. The proximal or base system achieves three degrees of freedom. The distal system, the hyperflexible component adds two more degrees of freedom. The base system is a conventional multiarm robot with constrained joints. The distal system is hollow core and has multiple elements, each of which can be separately actuated. The prototype has five individual parts, each of which can be actuated individually and also in combination. The hyperflexible component resembles an elephant trunk and moves the camera which is the payload of the device.

The elements of the hyperflexible system were built homogenously from 3D printed ABS plastic parts, and each of the printed elements was designed to achieve two degrees of freedom in the form of pitch and yaw movements. 
Each element has head and leg terminals. Multiple DC motors run both the divisions. The designed prototype consists of five such links and controlled by 18 DC motors. The motors are DC motors, lightweight $200 \mathrm{~g}, 6 \mathrm{~V}$ with driving current of $100 \mathrm{~mA}$, and a maximum speed of $500 \mathrm{rpm}$. They are assembled in a separate module. The $8 \times 8 \times 8 \mathrm{cc}$ DC motor size was suitable given the size constraints on the holding module. The payload comprising a camera does not require a high load current. The motors are all fixed at the base of the links and are individually connected to dedicated motors. The range of motion is limited to 15 degrees on each direction for an individual link. The entire distal system offers a maximum of 75 degrees bend. The elements are repeatable. Thus, by extending the links, and DC motors, the range of motion can be further enhanced. The DC motors are positioned along a spiral encompassing the holder, to allow undisturbed movement. A camera with a diameter of $5 \mathrm{~mm}$ and 2-megapixel resolution is mounted to the distalmost element to capture the probe's eye view. As a whole, the device described earlier is intended to perform translational, lateral, and angular movements. The display system in the current version is separate and is based on a dedicated laptop computer screen. A simple user interface is used to control the robot movement. The commands consist of six controls, namely, left, right, front, rear, up, and down, which is integrated into the system close to the surgeon user.

The device function has been tested in performing cadaver brain white fiber dissections. The cadaver brain setting resembles the actual neurosurgical operating field. The device has been tested in continuous operation for approximately 6 hours which is about the duration of a complex neurosurgical procedure. Though apparent limitations in terms of lighting, camera functions were present, dissection could be performed using the device. The data collected are novel and provide a unique perspective of a surgeon's skills (-Figs. 1, 2).

\section{Discussion}

Prof. Yasargil popularized the counterbalance system for neurosurgical microscopes and the technology for neurosurgical microscopy has stood the test of time. The ability of the counterbalance microscope to achieve stable userdetermined spatial configuration is unsurpassed. The devices in the course of evolution have changed little, a testimony to the engineering genius of the designers.

SARL focuses on various aspects of surgical robotics for applications in neurosurgery. Mythri is the first working product of the hyperflexible robotics program. The other focus of the group includes target systems, autonomous robotics, and surgical data science. The operating microscope is a product level spinoff of the project. The device has several novel features. It is an example of a serial robot wherein two independent robotic systems are assembled to achieve the desired result of spatial stability and maneuverability. The hyperflexible component has two degrees of freedom at each joint (between elements) which forms the technical novelty of the system. The design is simple and easily replicable.

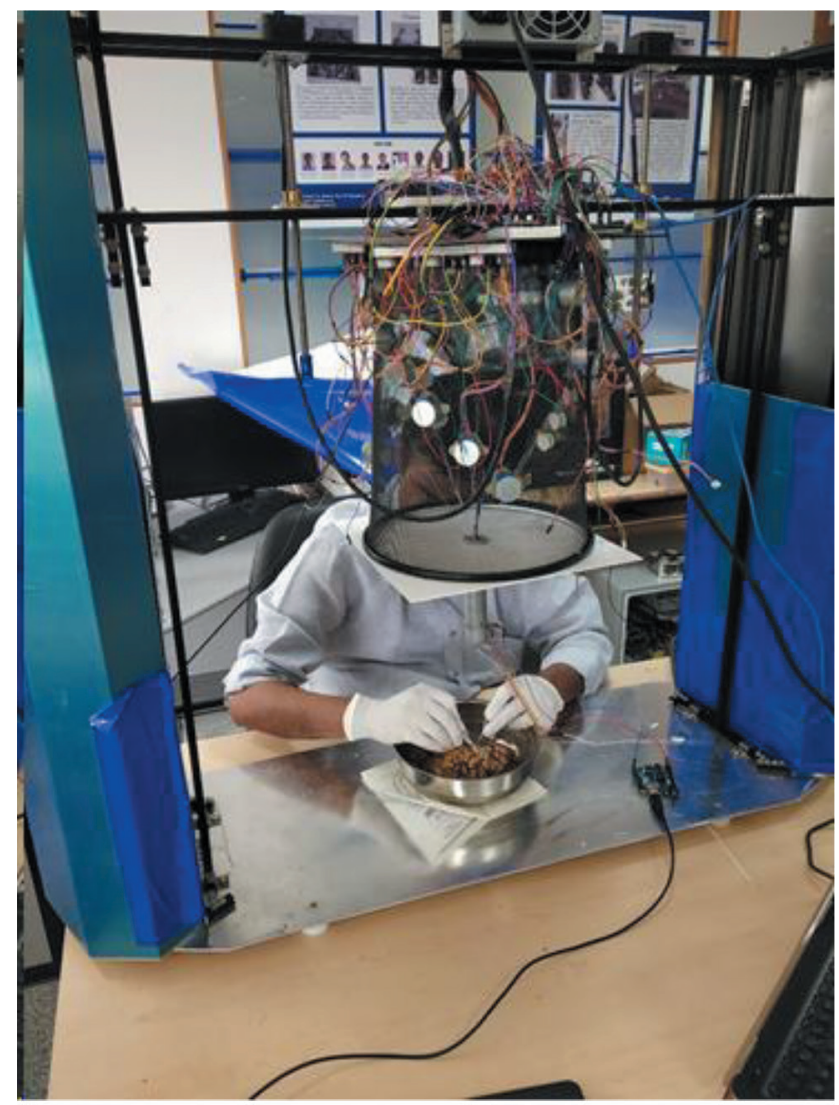

Fig. 1 Picture of the neurosurgical microscope assembly.

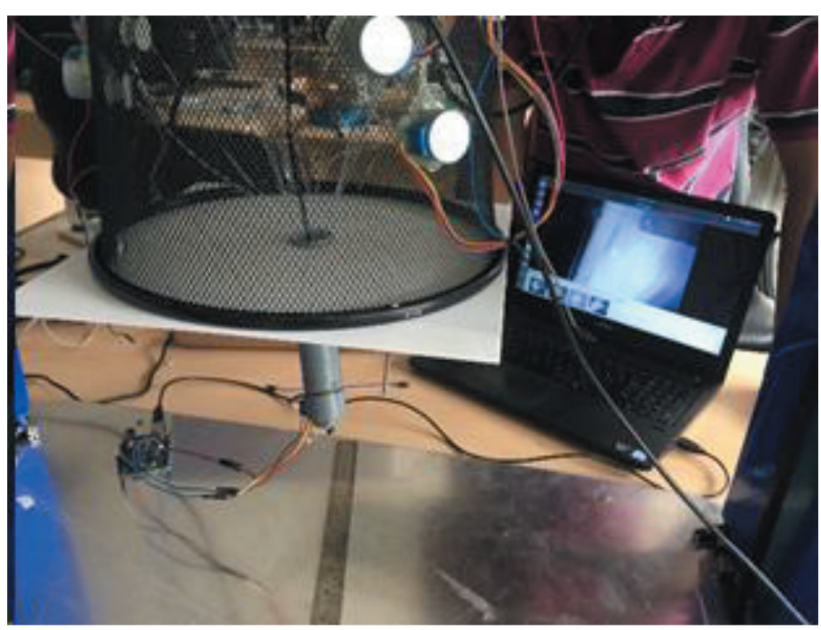

Fig. 2 Picture showing the probe of the surgical microscope with the video feed provided in the laptop.

The design incorporates off-the-shelf components, thus reducing the price of the device considerably. A microscope which is affordable, contemporary, and compact is required for our vast country where availability of high-end medical/ surgical devices is generally poor.

\section{Conclusion}

The development of Mythri 1.0 is a landmark development for Indian surgical robotics. The development proves 
the ability of Indian researchers to develop a world-class product with contemporary features. The initiative is expected to spur further growth in the area of surgical robotics in the country.

\section{Conflict of Interest}

V. V. reports a patent A flexible surgical device pending. A.R.V. reports a patent A hyperflexible surgical device pending. M.R. reports a patent A hyperflexible surgical device pending. K.A. reports a patent IPO application number TEMP/E-1/25142/2017-CHE pending.

\section{References}

1 Smith JA, Jivraj J, Wong R, Yang V. 30 Years of neurosurgical robots: review and trends for manipulators and associated navigational systems. Ann Biomed Eng 2016;44(4):836-846

2 Uluç K, Kujoth GC, Başkaya MK. Operating microscopes: past, present, and future. Neurosurg Focus 2009;27(3):E4

3 Yasargil MG, Microneurosurgery, Volume I: Microsurgical Anatomy of the Basal Cisterns and Vessels of the Brain, Diagnostic Studies, General Operative Techniques and Pathological Considerations of the Intracranial Aneurysms. Stuttgart, New York: Georg Thieme;1984:208

4 Belykh EG, Zhao X, Cavallo C, et al. Laboratory evaluation of a robotic operative microscope - visualization platform for neurosurgery. Cureus 2018;10(7):e3072

5 Krishnan KG, Schöller K, Uhl E. Application of a compact high-definition exoscope for illumination and magnification in high-precision surgical procedures. World Neurosurg 2017;97:652-660

6 Rossini Z, Cardia A, Milani D, Lasio GB, Fornari M, D’Angelo V. VITOM 3D: preliminary experience in cranial surgery. World Neurosurg 2017;107:663-668

7 Beez T, Munoz-Bendix C, Beseoglu K, Steiger H-J, Ahmadi SA. First clinical applications of a high-definition three-dimensional exoscope in pediatric neurosurgery. Cureus 2018;10(1):e2108

8 Ricciardi L, Chaichana KL, Cardia A, et al. The exoscope in neurosurgery: an innovative "point of view". A systematic review of the technical, surgical and educational aspects. World Neurosurg 2019;124:136-144

9 Takahashi S, Toda M, Nishimoto M, et al. Pros and cons of using

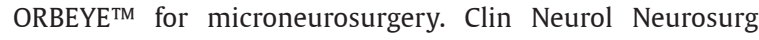
2018;174(September) :57-62

10 Klinger DR, Reinard KA, Ajayi OO. Delashaw JB Jr. Microsurgical clipping of an anterior communicating artery aneurysm using a novel robotic visualization tool in lieu of the binocular operating microscope: operative video. Oper Neurosurg (Hagerstown) 2018;14(1):26-28

11 Nishiyama K. From exoscope into the next generation. J Korean Neurosurg Soc 2017;60(3):289-293

12 Murai Y, Sato S, Yui K, et al. Preliminary clinical microneurosurgical experience with the 4K3-dimensional microvideoscope (ORBEYE) system for microneurological surgery: observation study. Oper Neurosurg (Hagerstown) 2019;16(6): 707-716

13 Vasefi F, MacKinnon N, Farkas DL, Kateb B. Review of the potential of optical technologies for cancer diagnosis in neurosurgery: a step toward intraoperative neurophotonics. Neurophotonics 2017;4(1):011010

14 Khalessi AA, Rahme R, Rennert RC, et al. First-in-man clinical experience using a high-definition 3-dimensional exoscope system for microneurosurgery. Oper Neurosurg (Hagerstown) 2019;16(6):717-725

15 Sato T, Bakhit MS, Suzuki K, et al. Utility and safety of a novel surgical microscope laser light source. PLoS One 2018;13(2):e0192112

16 Antoni S-T, Sonnenburg C, Saathoff T, Schlaefer A. Feasibility of interactive gesture control of a robotic microscope. Curr Dir Biomed Eng 2015;1(1):164-167

17 Pitskhelauri DI, Konovalov AN, Shekutev GA, et al. A novel device for hands-free positioning and adjustment of the surgical microscope. J Neurosurg 2014;121(1):161-164

18 Di Ieva A, Tam M, Tschabitscher M, Cusimano MD. A journey into the technical evolution of neuroendoscopy. World Neurosurg 2014;82(6):e777-e789

19 Friedman GN, Grannan BL, Nahed BV, Codd PJ. Initial experience with high-definition camera-on-a-chip flexible endoscopy for intraventricular neurosurgery. World Neurosurg 2015;84(6):2053-2058

20 Gaab MR. Instrumentation: endoscopes and equipment. World Neurosurg 2013;79(2, Suppl):14.e11-14.e21

21 Contreras López WO, Navarro PA, Crispin S. Intraoperative clinical application of augmented reality in neurosurgery: a systematic review. Clin Neurol Neurosurg 2019;177(177):6-11

22 Incekara F, Smits M, Dirven C, Vincent A. Clinical feasibility of a wearable mixed-reality device in neurosurgery. World Neurosurg 2018;118:e422-e427

23 Cutolo F, Meola A, Carbone M, et al. A new head-mounted display-based augmented reality system in neurosurgical oncology: a study on phantom. Comput Assist Surg (Abingdon) 2017;22(1):39-53

24 Guha D, Alotaibi NM, Nguyen N, Gupta S, McFaul C, Yang VXD. Augmented reality in neurosurgery: a review of current concepts and emerging applications. Can J Neurol Sci 2017;44(3):235-245 